\title{
Transvaginal electrical stimulation with surface-EMG biofeedback in managing stress urinary incontinence in women of premenopausal age: a double-blind, placebo-controlled, randomized clinical trial
}

\author{
Robert Terlikowski • Bozena Dobrzycka • \\ Maciej Kinalski • Anna Kuryliszyn-Moskal • \\ Slawomir J. Terlikowski
}

Received: 19 September 2012 / Accepted: 9 February 2013 / Published online: 27 February 2013

(C) The Author(s) 2013. This article is published with open access at Springerlink.com

\begin{abstract}
Introduction and hypothesis The aim of this study was to evaluate the results of conservative treatment of urodynamic stress urinary incontinence (SUI) using transvaginal electrical stimulation with surface-electromyography-assisted biofeedback (TVES + sEMG) in women of premenopausal age.

Methods One hundred and two patients with SUI were divided into two groups: active $(n=68)$ and placebo $(n=34)$ TVES + sEMG. The treatment lasted for 8 weeks and consisted of two sessions per day. Women were evaluated before and after the intervention by pad test, voiding diary, urodynamic test, and the Incontinence Quality of Life Questionnaire (I-QOL).

Results Mean urinary leakage on a standard pad test at the end of 8th week was significantly lower in the active than the placebo group $(19.5 \pm 13.6$ vs. $39.8 \pm 28.5)$. Mean urinary leakage on a 24 -h pad test was significantly reduced in the active group at the end of 8th and 16th weeks compared with the placebo group $(8.2 \pm 14.8$ vs. $14.6 \pm 18.9$ and $6.1 \pm$ 11.4 vs. $18.2 \pm 20.8$, respectively). There was also a significant improvement in muscle strength as measured by the
\end{abstract}

R. Terlikowski $\cdot$ A. Kuryliszyn-Moskal

Department of Rehabilitation, Medical University of Bialystok,

Bialystok, Poland

M. Kinalski

Department of Gynaecology, District General Hospital in

Bialystok, Bialystok, Poland

B. Dobrzycka $\cdot$ S. J. Terlikowski $(\bowtie)$

Department of Obstetrics, Gynaecology and Maternity Care

Medical University of Bialystok, ul. Szpitalna 37,

15-295 Bialystok, Poland

e-mail: sterlikowski@gmail.com
Oxford scale in the active vs the placebo group after 8 and 16 weeks (4.2 vs 2.6 and 4.1 vs 2.7 , respectively). No significant difference was found between groups in urodynamic data before and after treatment. At the end of 8th week, the mean I-QOL score in the active vs the placebo group was $78.2 \pm 17.9$ vs $55.9 \pm 14.2$, respectively, and at the end of 16 th week $80.8 \pm 24.1$ vs. $50.6 \pm 14.9$, respectively. Conclusion Our study showed that TVES + sEMG is a trustworthy method of treatment in premenopausal women with SUI; however, its reliability needs to be established.

Keywords Female stress urinary incontinence . Premenopausal age · Transvaginal electrical stimulation . Surface electromyography $\cdot$ Biofeedback

\section{Introduction}

Urinary incontinence (UI) affects $10-30 \%$ of women aged 15-64 years. Stress urinary incontinence (SUI) is the most common type of UI and is manifested by uncontrolled loss of urine due to physical exertion. The predisposing risk factors include attenuation of connective-tissue elasticity, vaginal delivery, obesity, hard physical work, and being elderly [1]. There are two types of commonly used SUI outcome measures: objective [cough stress test, pad test, and urodynamic evaluation) and subjective (patient self-assessment, validated questionnaires, voiding diaries, patient satisfaction, and quality of life (QOL)] [2]. A number of treatments for SUI are available, with the primary aim of reducing the occurrence of incontinence episodes and therefore limiting the impact of the disorder on an individual's QOL [3]. 
Physical therapies involving pelvic floor muscle training with or without other treatments, such as vaginal cones, biofeedback, and electrical stimulation (ES), are the core conservative and preventive treatments. Most studies present effect rates as combined cure and symptom improvement rates, mostly counting those women who improved by $\geq 50 \%$ on the primary outcome measure of effect. Short-term effect rates may exceed $70 \%$, whereas long-term effect rates may reach $50 \%$ when adherence is maintained [4, 5]. Many authors report that symptoms returned or increased after therapy had ended and concluded that declining adherence levels were probably the most important cause for declining long-term effect rates [6-8].

ES is a conservative treatment option described more than 50 years ago [9]. The mechanism of action of ES was initially investigated in animal models, where it caused bladder relaxation by inhibiting the parasympathetic motor neurones. Other studies showed that ES causes contractions of the pelvic floor, increasing the number of muscle fibers with rapid contraction that are responsible for continence in situations of stress [10].

Transvaginal electrical stimulation (TVES) is the nonpainful application of electrical current using a sensor with electrodes placed in the vagina to directly stimulate the pelvic floor muscles to contract and relax. Stimulation may help strengthen the muscles and ease SUI and can also help control urgency by acting on the nerves and decreasing bladder irritability $[11,12]$. The ideal electrical parameters for pelvic floor stimulation have not yet been well established. The few randomized clinical trials to date advocate using $20-50 \mathrm{~Hz}$ for SUI. The equipment is used transvaginally in daily sessions for $15-30 \mathrm{~min}$ at the maximum intensity individually tolerated for a variable period of 4-12 weeks $[13,14]$. The results of randomized controlled trials evaluating the effect of (ES) to treat SUI are conflicting. There is a need for more randomized controlled trials with sufficient sample sizes; use of sensitive, reproducible, and valid outcome measures; and optimal stimulation parameters [15].

Surface EMG (sEMG) is one method used to evaluate SUI treatment outcome by recording the change in voltage over the muscle fiber membrane that initiates the contraction. Control of the muscle contraction is mediated via nerve impulses in the motor nerves. For skeletal muscles, there is generally a relationship between EMG activity and force, which means that higher activity levels correspond to an increase in force [16].

Biofeedback involves using electrodes to transduce muscle potentials into auditory or visual signals; patients learn to increase or decrease voluntary muscle activity. Biofeedback is useful to promote correct control of contraction and visualization, because many women cannot at first contract their pelvic floor muscles and require some type of motivation. Biofeedback treatments are safe and effective interventions that should be more readily available to patients as a first-line treatment for voiding dysfunction. Patient education may take time but has higher long-term success and makes the patient more responsible and less passive regarding their condition [17].

Although strong evidence suggests that TVES is effective for reducing SUI symptoms, data on adjunctive techniques are less consistent. Studies are significantly heterogeneous in methodology and follow-up, making conclusions difficult. Thus, the aim of this study was to evaluate the results of conservative SUI treatment using TVES with sEMGassisted biofeedback in women of premenopausal age.

\section{Materials and methods}

One hundred and two women with urodynamic SUI who underwent treatment at our departments from January 2008 to April 2012 were enrolled in this study. Standardized assessment was performed at enrolment, taking into consideration a comprehensive urogynecological history, urodynamic assessment including uroflowmetry and cystometry, bacteriological examination, and pad tests. The protocol was previously approved by the Bioethical Committee of the Medical University of Bialystok (R-I-002/143/2009), and all women gave their written consents.

Major exclusion factors were patients with chronic degenerative diseases that would affect muscular and nerve tissues, presence of any degree of pelvic organ prolapse (POP), active or recurrent urinary tract infections (UTI), vulvovaginitis, atrophic vaginitis, diabetes mellitus, neurological disease, psychiatric illness, use of medication affecting micturition, history of surgical or pharmaceutical treatment of SUI, chronic debilitating disease such as renal failure, and those with cardiac pacemakers. We also excluded patients with intrinsic sphincteric deficiencies identified by the Valsalva leak-point pressure $\leq 60 \mathrm{cmH}_{2} 0$ measurement in the sitting position with a volume of $250 \mathrm{ml}$ in the bladder and/or a urethral closure pressure $\leq 20 \mathrm{~cm} \mathrm{H}_{2} 0$ in the sitting position at maximum cystometric capacity.

Participants were taught skills and strategies for preventing incontinence and suppressing urge. This included education about normal bladder control, lifestyle interventions such as weight reduction, relieving constipation, smoking cessation, caffeine reduction, fluid management, wearing nonrestrictive and easily removed clothing, reducing emotional stress, and correcting faulty habit patterns of frequent urination by suggesting distraction and avoidance techniques. Advice on good voiding position was also provided. In addition, an information booklet was provided to reinforce this information.

The severity of UI can be assessed by various methods, such as a urinary symptoms questionnaire, frequency/volume chart (bladder diary), QOL questionnaire, or pad tests. A 7- 
day bladder diary provides the means for assessing voiding frequency and volume, together with the frequency of leaks at 0,8 , and 16 weeks.

During initial examination, a POP and pelvic floor dysfunction assessment was performed, as previously described, with the patient supine and after voiding [18]. Assessment of pelvic floor muscle strength was performed with vaginal palpation; the woman in the lithotomy position used one finger with the two distal phalanges inside the introitus vagina. One gynecologist per department should examine and palpate the external and internal vaginal and rectal muscles for function, pain, strength, coordination, and endurance for standardizing the methodology. The effort was graded with the Oxford score ( 0 nil; 1 flicker; 2 weak; 3 medium, with slight lift of the examiners finger and no resistance; 4 strong, sufficient to elevate the examiner's finger against light resistance; 5 very strong, sufficient to elevate the examiner's finger against strong resistance) [5].

Next, the bladder was drained to obtain a postvoid residual, and the s bladder was then retrograde filled to a volume of $300 \mathrm{ml}$ or maximal capacity (if that occurred prior to $300 \mathrm{ml}$ ). The patient was then cued to cough and perform a Valsalva maneuver while supine. If no urine leakage was observed, the patient was asked to perform the same maneuvers while standing. The cough stress test was considered positive if any leakage was noted with cough or Valsalva [19].

Uroflowmetry (Ellipse Andromeda System, Germany) was performed on all participants. Each participant was asked to attend the session with a comfortably full bladder to perform a free-flow uroflowmetry. In order to carry out the test, it was necessary to gain an artificial standardized fill of $250 \mathrm{ml}$ using a size-12 Foley urethral catheter at a maximum filling rate of $20 \mathrm{ml}$ per minute. After emptying the patient's bladder with a catheter, the 20-min pad test by infusion of $250 \mathrm{ml}$ distilled water in the bladder was performed after the urodynamic study. Each patient returned to a standing position, with a preweighed perineal pad placed inside the underwear. Each patient was asked to cough ten times, bear down ten times, do ten deep knee bends, jump up and down in place ten times, wash her hands under cold water for $1 \mathrm{~min}$, walk up and down five stairs ten times, walk in the hall for $10 \mathrm{~min}$, and then return for removal of the pad (Bella Sp. z o.o., Torun, Poland). The pad was then weighed, and the net weight was calculated by subtracting the original dry weight to achieve a measure of the total urine lost during the 20-min exercise. A pad weight result was considered positive if a leakage $>1 \mathrm{~g}$ was noted; pads were weighed on a precision laboratory balance Acculab VI-1200 (Bradford, MA, USA) [20].

Patients with urodynamically confirmed SUI were randomized using a computer-generated random sequence given in sealed envelopes to patients in group 1 [active $(n=68)$ ] or group 2 [placebo $(n=34)$ ] TVES with sEMG-assisted biofeedback. In a previous study with 24 patients, the final sample size was calculated as 102 patients for a power of $80 \%$ and a $2: 1$ ratio (68 and 34 , respectively). Patient distribution was chosen to better evaluate the proposed technique. One hundred and nineteen individuals were screened for eligibility: 107 were eligible, five of whom withdrew before completion of assessments. Thus, 102 were recruited. Group assignment was enclosed in sequentially numbered, sealed envelopes by a person not involved in the study. The physiotherapist and physician carrying out the assessment were unaware of which treatment group the patient was in. To minimize the likelihood of assessor bias, participants were asked not to discuss their treatment and/or reveal any information on group allocation to the principal investigator doing the assessments. Participants were scheduled to attend clinic visits. All interventions were performed by gynecologists and competent physiotherapists. Screening, outcome assessments, and weekly visits were carried out at hospital sites offering identical facilities.

In group 1 TVES, was provided with the addition of sEMG biofeedback. EMG records muscle bioelectrical activity and measures electrical correlation of the muscle contraction. Vaginal and surface electrodes can be used to provide an overview of the contraction and relaxation phases of pelvic floor muscle activity. EMG biofeedback assessment used a NeuroTrac ${ }^{\text {TM }}$ ETS unit (Verity Medical Ltd, Hampshire, UK). A vaginal electrode, VeriProbe (Verity Medical), was applied according to the manufacturer's instructions with Zelpol lubricating couplant (Centrum Medicum, Poland), and stimulation parameters and patient-acceptable sensitivity thresholds were determined. The device combines biofeedback and (ES), with effective monitoring of compliance with treatment and performance. Patient position, accuracy of electrode placement, exact warmup period, and time of day were all recorded. The regimen included a warmup of five contractions and five relaxations, followed by a contraction/relaxation assessment. Participants were encouraged to selectively contract and relax their pelvic floor muscles with the assistance of visual and auditory feedback.

Patients in group 1 were provided with active TVES with sEMG. Parameters of muscle stimulation were adapted for each participant: frequency ranged from 10 to $40 \mathrm{~Hz}$, impulse width from 200 to $250 \mu \mathrm{s}$, and runtime/decontraction in configuration of $15 \mathrm{~s} / 30 \mathrm{~s}$ for $20 \mathrm{~min}$. The treatment lasted for 8 weeks and was performed twice a day. The introduction took place in the clinic, and the actual treatment was performed by patients at home, with a gradual increase to a daily maximum of $40 \mathrm{~min}$. At each weekly visit, compliance was monitored by means of a concealed button on the Neurotrac ${ }^{\mathrm{TM}}$ ETS unit, and the unit was reprogrammed weekly, as appropriate.

Participants in group 2 were provided with a placebo set to parameters proven to have no physiological effect. The same type of electrode and hand-held unit as described for TVES 
with sEMG biofeedback was used in the clinic and for home application. Preset parameters were a frequency of $2 \mathrm{~Hz}$, a pulse width of $50 \mu \mathrm{s}, 2 \mathrm{~s}$ of stimulation, and $60 \mathrm{~s}$ of no stimulation, with a ramp of $8 \mathrm{~s}$. As with group 1, the introduction took place in the clinic, and patients used issued devices at home, with a gradual increase to a daily maximum of $40 \mathrm{~min}$. Treatment continued until participants had completed 8 weeks with the assigned device. At this point, a posttreatment assessment identical to the intake assessment was performed. Participants underwent three 24-h pad tests using Bella Normal sanitary pads. For each test (week 0,8 , and 16), five pads each contained in their own sealable plastic bag were weighed and given to participants. Women were asked to wear the pads for
$24 \mathrm{~h}$ : one pad at a time for a maximum of $6 \mathrm{~h}$ except for the pad worn during the night's sleep, which could be worn all night. After usage, the pad, its wrapper, and adhesive strips were returned to their plastic bag and sealed to prevent evaporation. The two pad tests were to be completed within a week and returned to the clinic within the following week prior to initiation of the new treatment. The pads were reweighed, and the urine lost during each 24-h period was calculated. If the wrapper and adhesive strips were not returned, pad weight results were adjusted to account for their weight. Pre- and postpad weights were determined using the same beam balance scales with accuracy to $0.1 \mathrm{~g}$. The 24 -h pad test was considered positive if a weight exceeded $8 \mathrm{~g}[19,21]$.
Fig. 1 Recruitment and progress of participants throughout the trial

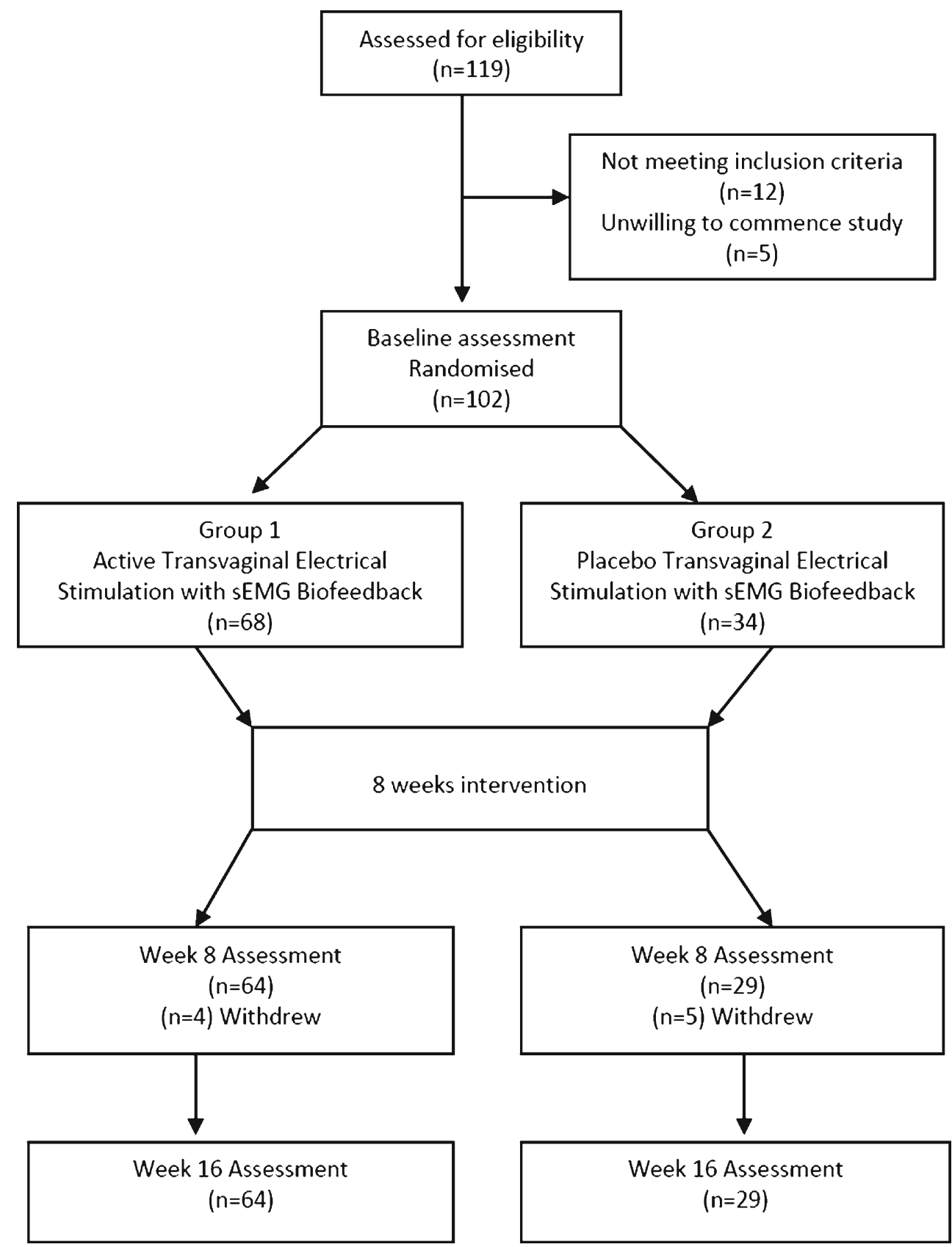


Table 1 Selected demographic and baseline clinical characteristics for each group

Data presented as mean \pm standard deviation, data in parenthesis are percentages. Denotes overall comparison among groups using chi-square test and independent samples $t$ test

\begin{tabular}{llll}
\hline Variables & Group $1 n=64$ & Group $2 n=29$ & $P$ value \\
\hline Age & $46.9 \pm 6.8$ & $45.6 \pm 7.9$ & 0.591 \\
Body mass index & $24.9 \pm 3.7$ & $25.1 \pm 2.8$ & 0.197 \\
Smoker (\%) & $7(10.9)$ & $4(13.8)$ & 0.355 \\
Durations of symptoms (year) & $6.0 \pm 3.9$ & $5.9 \pm 4.9$ & 0.260 \\
Pregnancy & $3.1 \pm 2.1$ & $3.8 \pm 1.8$ & 0.750 \\
Vaginal delivery & $3.5 \pm 2.6$ & $3.1 \pm 2.7$ & 0.902 \\
Cesarean delivery & $0.5 \pm 0.4$ & $0.5 \pm 0.9$ & 0.432 \\
\hline
\end{tabular}

All study participants were also asked to complete the validated Incontinence Quality of Life (I-QOL) questionnaire containing one section. A score of 100 represented the best possible QOL, and 0 represented the worst possible QOL. Valid and reliable outcome measures were recorded at weeks 0 (baseline), 8 , and 16 . The I-QOL contains 22 negatively framed items, each with a five-point Likert-type response scale (1 extremely, 2 quite a bit, 3 moderately, 4 a little, 5 not at all). The I-QOL was scored according to the instructions [22]. In addition to yielding a total score, the IQOL consists of the following three domain scores: avoidance and limiting behaviors (eight items), psychosocial impacts (nine items), and social embarrassment (five items). The I-QOL total and subscale sum scores are transformed onto a $0-100$ scale for greater interpretability, with the higher scores representing greater QOL. Once the I-QOL was scored and plotted, variations in mean scores were noted across the language versions [23].

Statistical analysis was performed using Statistica software version 9.0PL (StatSoft, Inc., StatSoft Polska Sp. z o.o., Poland). A chi-square test was used to evaluate the relationship between categorical variables. Fisher's exact test was used to determine significance between groups. The MannWhitney $U$ test was used for comparison of continuous variables between groups. Comparisons within each group were carried out using the Wilcoxon signed rank test. A $p$ value $<$ 0.05 was considered statistically significant.

\section{Results}

One hundred and nineteen individuals with SUI were screened (Fig. 1). Twelve failed to meet the inclusion/exclusion criteria, and a further five were unwilling to take part in the study. There were no statistically significant differences detected in demographic data and clinical characteristics between groups (Table 1).

During the 8 weeks of active treatment, four participants in group 1 withdrew completely because the protocol was found to be too demanding $(n=2)$ or they were unable to use the stimulator at home $(n=2)$. Five participant in tgroup 2 discontinued because they used other treatments during the trial $(n=3)$, change of work $(n=1)$, and death in the family $(n=1)$. The overall scores on the I-QOL are given in Table 2 . At baseline the mean I-QOL score in group 1 was $61.8 \pm 13.4$ and in group $266.2 \pm 23.8(p=0.241)$. At the end of 8 th week, the mean I-QOL score in group 1 was $78.2 \pm 17.9$ and in group $255.9 \pm 14.2(p=0.004)$; at the end of 16 th week, scores were $80.8 \pm 24.1$ vs. $50.6 \pm 14.9$, respectively $(p<0.001)$. In group 1 , there was a significant reduction in subjective perception of the impact of incontinence during the treatment period.

At baseline, a standard 20-min pad tests showed no significant differences between groups 1 and $2(25.6 \pm 27.1$ vs. $27.9 \pm 29.1 ; p=0.310$ ). Mean urinary leakage on a standard pad test at the end of 8th week was significantly lower in the group 1 compared with the group $2(19.5 \pm 13.6$ vs.
Table 2 Subjective and objective variables before and after treatment

Data presented as mean \pm standard deviation. Denotes overall comparison between groups using Mann-Whitney $U$ test

\begin{tabular}{llll}
\hline Variables & Group 1 $n=64$ & Group 2 $n=29$ & $P$ value \\
\hline I-QOL (baseline) & $61.8( \pm 13.4)$ & $66.2( \pm 23.8)$ & 0.241 \\
I-QOL (8 weeks) & $78.2( \pm 17.9)$ & $55.9( \pm 14.2)$ & 0.004 \\
I-QOL (16 weeks) & $80.8( \pm 24.1)$ & $50.6( \pm 14.9)$ & $<0.001$ \\
20-min pad test (g) (baseline) & $25.6( \pm 27.1)$ & $27.9( \pm 29.1)$ & 0.310 \\
20-min pad test (g) (8 weeks) & $19.5( \pm 13.6)$ & $39.8( \pm 28.5)$ & 0.003 \\
24-h pad test (g) (baseline) & $12.4( \pm 18.2)$ & $13.9( \pm 16.3)$ & 0.344 \\
24-h pad test (g) (8 weeks) & $8.2( \pm 14.8)$ & $14.6( \pm 18.9)$ & 0.004 \\
24-h pad test (g) (16 weeks) & $6.1( \pm 11.4)$ & $18.2( \pm 20.8)$ & 0.002 \\
Oxford score (baseline) & 2.9 & 2.8 & 0.339 \\
Oxford score (8 weeks) & 4.2 & 2.6 & $<0.001$ \\
Oxford score (16 weeks) & 4.1 & 2.7 & $<0.001$ \\
\hline
\end{tabular}


Table 3 Seven-day voiding diary before and after treatment
Data presented as mean \pm standard deviation. $P$ value determined by Wilcoxon signed rank test.

\begin{tabular}{lllr}
\hline Variables & Group 1 n=64 & Group 2 $n=29$ & $P$ value \\
\hline Voiding frequency(baseline) & $48.4( \pm 19.8)$ & $46.8( \pm 16.8)$ & 0.341 \\
Voiding frequency (8 weeks) & $47.2( \pm 12.4)$ & $48.6( \pm 18.6)$ & 0.224 \\
Voiding frequency (16 weeks) & $48.2( \pm 16.8)$ & $49.2( \pm 19.4)$ & 0.327 \\
Frequency of urine loss (baseline) & $16.4( \pm 18.4)$ & $14.8( \pm 16.2)$ & 0.311 \\
Frequency of urine loss (8 weeks) & $6.7( \pm 5.3)$ & $16.2( \pm 14.7)$ & $<0.001$ \\
Frequency of urine loss (16 weeks) & $6.2( \pm 5.8)$ & $17.2( \pm 15.6)$ & $<0.001$ \\
Occurrence of nocturia (baseline) & $12.4( \pm 10.7)$ & $13.8( \pm 11.4)$ & 0.283 \\
Occurrence of nocturia (8 weeks) & $6.7( \pm 4.7)$ & $14.6( \pm 12.4)$ & 0.012 \\
Occurrence of nocturia (16 weeks) & $6.8( \pm 4.9)$ & $15.4( \pm 12.9)$ & 0.002 \\
No. of pads used (baseline) & $9.2( \pm 7.4)$ & $8.7( \pm 6.8)$ & 0.324 \\
No. of pads used (8 weeks) & $3.4( \pm 4.2)$ & $9.2( \pm 7.6)$ & 0.022 \\
No. of pads used (16 weeks) & $3.8( \pm 4.8)$ & $9.1( \pm 6.9)$ & 0.024 \\
\hline
\end{tabular}

$39.8 \pm 28.5 ; p=0.003)$. Mean urinary leakage on a $24-\mathrm{h}$ pad test was significantly reduced in group 1 at the end of weeks 8 and 16 compared with group 2 ( $8.2 \pm 14.8$ vs. 14.6 \pm 18.9 ; $p=0.004$ and $6.1 \pm 11.4$ vs. $18.2 \pm 20.8 ; p=0.002$, respectively). There was also a significant improvement in muscle strength as measured by the Oxford scale in group 1 compared with group 2 after 8 and 16 weeks (4.2 vs 2.6 and 4.1 vs 2.7, respectively; $p<0.001$ ) (Table 2 ). On the basis of the 7-day voiding-diary logs, we there was no change in voiding frequency between groups. The severity of urinary symptoms, such as frequency of urine loss, occurrence of nocturia, and number of pads used, significantly decreased in group 1 compared with group 2 at 8 and 16 weeks (Table 3 ).

No significant difference was found between groups in urodynamic data before and after treatment (Table 4).

Twenty-five women (39\%) were objectively cured on the standard pad test, and a cure was defined as $\leq 1 \mathrm{~g}$ of leakage with a standardized bladder volume. In group 2, objective cure was not achieved. Subjectively, 29 (45.3\%) of patients in group 1 considered themselves cured, 12 (18.7\%) improved, 17 (26.6\%) unchanged, and six (9.4\%) worse. In group 2, subjective cure was reported by two (6.9\%), improvement by four (13.8\%), no change by 14 (48.3\%), and worsening by nine $(31 \%)$. There were no side effects reported in group 2; in group 1, four participants reported smarting (three bleeding, one discomfort).

\section{Discussion}

For patients with SUI, urine leakage and loss of muscle strength are classified as impairments of body function, with significant socioeconomic and QOL impact. SUI imposes significant restrictions on patients in regards to social activities, travel, recreation, and personal relationships. Several conservative treatment options are available for managing
Table 4 Urodynamic data before and after treatment

Data presented as mean \pm standard deviation. Data in parenthesis are percentages. Denotes overall comparison among groups using Mann-Whitney $U$ test

\begin{tabular}{llll}
\hline Cystometry & Group 1 $n=64$ & Group $2 n=29$ & $P$ value \\
\hline Volume at first desire to void (ml) baseline & $134.6 \pm 51.6$ & $138.2 \pm 57.1$ & 0.911 \\
Volume at first desire to void (ml) 8 weeks & $149.6 \pm 69.3$ & $141.1 \pm 49.2$ & 0.07 \\
Maximum cystometric capacity (ml) baseline & $536.7 \pm 149.8$ & $528.2 \pm 149.8$ & 0.777 \\
Maximum cystometric capacity (ml) 8 weeks & $589.2 \pm 149.7$ & $487.2 \pm 111.9$ & 0.223 \\
Postmicturition residual (ml) baseline & $8.1 \pm 3.4$ & $10.8 \pm 5.1$ & 0.801 \\
Postmicturition residual (ml) 8 weeks & $5.1 \pm 4.0$ & $11.8 \pm 7.8$ & 0.693 \\
No. (\%) with negative stress test (urodynamic) 8 weeks & $13(20.3)$ & $2(6.9)$ & \\
Functional urethral length (cm) baseline & $2.8 \pm 0.9$ & $2.7 \pm 0.8$ & 0.466 \\
Functional urethral length (cm) 8 weeks & $2.6 \pm 0.5$ & $2.5 \pm 0.5$ & 0.560 \\
Maximum urethral closure pressure (cm $\mathrm{H}_{2} \mathrm{O}$ ) baseline & $53.8 \pm 8.2$ & $52.9 \pm 7.1$ & 0.579 \\
Maximum urethral closure pressure (cm $\left.\mathrm{H}_{2} \mathrm{O}\right) 8$ weeks & $55.9 \pm 6.9$ & $54.9 \pm 9.8$ & 0.518 \\
Maximum flow rate ml/s baseline & $29.4 \pm 9.1$ & $27.8 \pm 6.8$ & 0.714 \\
Maximum flow rate ml/s 8 weeks & $30.3 \pm 10.1$ & $28.4 \pm 9.2$ & 0.105 \\
Average flow rate ml/s baseline & $15.9 \pm 9.4$ & $16.1 \pm 9.5$ & 0.911 \\
Average flow rate ml/s 8 weeks & $17.5 \pm 8.2$ & $18.1 \pm 10.1$ & 0.188 \\
\hline
\end{tabular}


SUI $[24,25]$ : TVES is one nonsurgical approach. Its purpose is to increase urethral resistance by strengthening the pelvic floor musculature and, consequently, improving incontinence associated with stress. It is an effective and safe therapy, but results vary widely from study to study, even when treatment uses the same device. Comparative analyses among standard urodynamic parameters before and after several sessions of (ES) do not clearly explain the influence of the vaginal electrode on outcomes [26].

In our study, no significant difference was found between groups in urodynamic data before and after treatment. Therefore, as with Resplande et al. [26], we concluded that, at least acutely, (ES) did not modify some baseline cystometry parameters and did not modify the mechanical presence of the vaginal electrode when turned off. However, it is important to remember that the correlation between clinical improvement and urodynamic changes remains controversial [14].

In patients with SUI, pelvic floor (ES) is suggested to act at the muscular level by activating the pudendal nerve motor efferents and through reflex activation. Stimulation would promote successive contractions of the pelvic floor muscles, thus invigorating and strengthening such muscle fibers $[10$, 27]. Because the pudendal nerve is an efferent nerve for both the pelvic floor muscles and the external urethral sphincter, damage to it can generate weakness of the pelvic floor, resulting in POP, and relaxation of the external urethral sphincter, resulting in SUI [28].

Comparison between published studies of TVES is difficult because different therapies and different outcome variables are used. It is reported that there is strong evidence in favor of TVES or TVES with sEMG versus no treatment for SUI in women $[5,29]$. It is known that discontinuation of a program will result in loss of any therapeutic effect within 4-6 weeks, whereas continuation of daily treatment for 36 months is essential to preserve the achieved muscle strength [30].

Results of our study demonstrate that TVES with sEMG increased muscle strength most during the first 2 months of treatment and was well preserved at month 4 . This is in line with findings of pad tests and subjective I-QOL questionnaires. We found that this intervention is of great importance if used at the beginning of therapy and in the follow-up period and it is highly reproducible. It makes it easier for the physiotherapist to teach isolated pelvic floor muscles contraction, facilitates goal setting, and helps keep the patient highly motivated. We also show that compliance was homogeneous and satisfactory, probably due to the clear and consistent directions and the characteristics of the equipment. The subjective success rate of those therapies was approximately $45 \%$ and the objective (pad test) rate approximately $39 \%$. Treatment was very well tolerated, and few adverse events could be correlated with treatment. The significant improvements in QOL demonstrated in this study are important because they help us to understand the clinical relevance in alterations in pad tests and voiding diary. Such an outcome was expected, as by receiving visual feedback on the intensity and contraction waveform, patients have a chance to correct themselves. The addition of ES triggered by the pressure signal aims to create an association between the voluntary attempts to produce a contraction and an actual contraction that is guaranteed by electrical stimulus. In this study, a physiotherapist supervised all sessions in the clinic and increased the intensity of electrical impulses. This engagement was probably responsible for the high rate of compliance with treatment and the favorable outcomes based on subjective and objective measures.

QOL has become an important outcome measure in clinical trials dealing with SUI treatment. In our study, we observed a significant improvement in QOL of treated women after the intervention, particularly with regard to factors related to limitations (in physical and social activities) and to severity measures. Whereas QOL scores differed in some countries (i.e., lower scores in Poland and Slovakia, and higher scores in Greece and The Netherlands), I-QOL score component trends remained very similar. The differences are more than likely related to differences in population characteristics rather than measurement errors. Reliability, using Cronbach's alpha, was confirmed in all language versions, where high internal consistency was demonstrated by alpha values above 0.70 . Similarly, test-retest reliability showed the I-QOL to be stable over time, with intraclass correlation coefficient (ICC) values all greater than the recommended 0.70 [23].

In conclusion, this study shows that TVES with sEMG is a trustworthy method for treating premenopausal women with SUI; however, reliability needs to be established.

\section{Conflicts of interest None.}

Open Access This article is distributed under the terms of the Creative Commons Attribution License which permits any use, distribution, and reproduction in any medium, provided the original author(s) and the source are credited.

\section{References}

1. Sievert KD, Amend B, Toomey PA et al (2012) Can we prevent incontinence? ICI-RS 2011. Neurourol Urodyn 31(3):390-399

2. Latthe PM, Foon R, Khan K (2008) Nonsurgical treatment of stress urinary incontinence (SUI): grading of evidence in systematic reviews. BJOG 115(4):435-444

3. Coyne KS, Kvasz M, Ireland AM et al (2012) Urinary incontinence and its relationship to mental health and health-related quality of life in men and women in Sweden, the United Kingdom, and the United States. Eur Urol 61(1):88-95

4. Liebergall-Wischnitzer M, Hochner-Celnikier D, Lavy Y et al (2009) Randomized trial of circular muscle versus pelvic floor training for stress urinary incontinence in women. J Womens Health (Larchmt) 18(3):377-385 
5. Dannecker C, Wolf V, Raab R et al (2005) EMG-biofeedback assisted pelvic floor muscle training is an effective therapy of stress urinary or mixed incontinence: a 7-year experience with 390 patients. Arch Gynecol Obstet 273(2):93-97

6. Imamura M, Abrams P, Bain C et al (2010) Systematic review and economic modelling of the effectiveness and cost-effectiveness of non-surgical treatments for women with stress urinary incontinence. Health Technol Assess 14(40):1-188

7. Labrie J, van der Graaf Y, Buskens E et al (2009) Protocol for Physiotherapy Or TVT Randomised Efficacy Trial (PORTRET): a multicentre randomised controlled trial to assess the costeffectiveness of the tension free vaginal tape versus pelvic floor muscle training in women with symptomatic moderate to severe stress urinary incontinence. BMC Womens Health 9:24

8. Bø K, Hilde G (2012) Does it work in the long term?-A systematic review on pelvic floor muscle training for female stress urinary incontinence. Neurourol Urodyn. doi:10.1002/nau.22292

9. Caldwell KP (1963) The electrical control of sphincter incompetence. Lancet 2(7300):174-175

10. Fall M, Lindström S (1991) Electrical stimulation. A physiologic approach to the treatment of urinary incontinence. Urol Clin North Am 18(2):393-407

11. Monga AK, Tracey MR, Subbaroyan J (2012) A systematic review of clinical studies of electrical stimulation for treatment of lower urinary tract dysfunction. Int Urogynecol J 23(8):993-1005

12. Schmidt AP, Sanches PR, Silva DP Jr et al (2009) A new pelvic muscle trainer for the treatment of urinary incontinence. Int $\mathrm{J}$ Gynaecol Obstet 105(3):218-222

13. Alves PG, Nunes FR, Guirro EC (2011) Comparison between two different neuromuscular electrical stimulation protocols for the treatment of female stress urinary incontinence: a randomized controlled trial. Rev Bras Fisioter 15(5):393-398

14. Castro RA, Arruda RM, Zanetti MR et al (2008) Single-blind, randomized, controlled trial of pelvic floor muscle training, electrical stimulation, vaginal cones, and no active treatment in the management of stress urinary incontinence. Clinics (Sao Paulo) 63(4):465-472

15. Bø K (1998) Effect of electrical stimulation on stress and urge urinary incontinence. Clinical outcome and practical recommendations based on randomized controlled trials. Acta Obstet Gynecol Scand Suppl 168:3-11

16. De Luca CJ (1997) The use of surface electromyography in biomechanics. J Appl Biomech 13:135-163
17. Herderschee R, Hay-Smith EJ, Herbison GP et al (2011) Feedback or biofeedback to augment pelvic floor muscle training for urinary incontinence in women. Cochrane Database Syst Rev (7): CD009252

18. Bump RC, Mattiasson A, Bø K et al (1996) The standardization of terminology of female pelvic organ prolapse and pelvic floor dysfunction. Am J Obstet Gynecol 175(1):10-17

19. Price DM, Noblett K (2012) Comparison of the cough stress test and 24-h pad test in the assessment of stress urinary incontinence. Int Urogynecol J 23(4):429-433

20. Wu WY, Sheu BC, Lin HH (2006) Comparison of 20-min pad test versus 1-h pad test in women with stress urinary incontinence. Urology 68(4):764-768

21. Painter V, Karantanis E, Moore KH (2012) Does patient activity level affect 24 -h pad test results in stress-incontinent women? Neurourol Urodyn 31(1):143-147

22. Patrick DL, Martin ML, Bushnell DM et al (1999) Quality of life of women with urinary incontinence: further development of the Incontinence Quality of Life Instrument (I-QOL). Urology 53:7176

23. Bushnell DM, Martin ML, Summers KH et al (2005) Quality of life of women with urinary incontinence: cross-cultural performance of 15 language versions of the I-QOL. Qual Life Res 14(8):1901-1913

24. Caruso DJ, Gomez CS, Gousse AE (2009) Medical management of stress urinary incontinence: is there a future? Curr Urol Rep 10(5):401-407

25. Holroyd-Leduc JM, Straus SE (2004) Management of urinary incontinence in women: scientific review. JAMA 291(8):986-995

26. Resplande J, Gholami S, Bruschini H et al (2003) Urodynamic changes induced by the intravaginal electrode during pelvic floor electrical stimulation. Neurourol Urodyn 22(1):24-28

27. Teague CT, Merrill DC (1977) Electric pelvic floor stimulation. Mechanism of action. Invest Urol 15(1):65-69

28. Chai TC, Steers WD (1997) Neurphysiology of micturition and continence in women. Int Urogynecol Urol 8:85-97

29. Weatherall M (1999) Biofeedback or pelvic floor muscle exercises for female genuine stress incontinence: a meta-analysis of trials identified in a systematic review. BJU Int 83(9):1015-1016

30. Gondin J, Guette M, Ballay Y et al (2006) Neural and muscular changes to detraining after electrostimulation training. Eur J Appl Physiol 97(2):165-173 